\title{
Effect of donepezil on Hcy level in serum of Alzheimer's disease patients and correlation analysis of Hcy and dyssomnia
}

\author{
XINXIN LIU ${ }^{1,2}$, JIEWEN ZHANG ${ }^{1}$, MINGRONG XIA ${ }^{1}$, JUNRAN LIU $^{1}$ and SHAN JIANG ${ }^{1}$ \\ ${ }^{1}$ Department of Neurology, Zhengzhou University People's Hospital, Zhengzhou, Henan 450003; \\ ${ }^{2}$ Department of Neurology, The First Affiliated Hospital of Henan University, Kaifeng, Henan 47500, P.R. China
}

Received May 22, 2018; Accepted November 20, 2018

DOI: $10.3892 /$ etm.2018.7071

\begin{abstract}
Effect of donepezil on the homocysteine (Hcy) level in serum of Alzheimer's disease (AD) patients and correlation between Hcy and dyssomnia was investigated. A retrospective analysis of $124 \mathrm{AD}$ patients in Zhengzhou University People's Hospital between January 2015 and October 2017 was performed, including 64 cases in the observation group and 60 cases in the control group. The control group was treated with folic acid, vitamin B12 and memantine hydrochloride tablet, and the observation group combined with donepezil on this basis, and both groups were treated for 4 months. The Hcy level before and after treatment was detected in the groups using ELISA method, dyssomnia score of patients was performed before and after treatment in the observation group according to Pittsburgh Sleep Quality Index (PSQI), and correlation analysis between the Hcy level before and after treatment and dyssomnia was performed in AD patients in the study group using Pearson's correlation analysis. The differences were statistically significant in the Hcy level before and after treatment in both groups $(\mathrm{P}<0.001)$. The Hcy level after treatment in the observation group was significantly lower than that in the control group $(\mathrm{P}<0.001)$. The dyssomnia score before treatment was higher that after treatment in the observation group $(\mathrm{P}<0.001)$. There was a positive correlation between the Hcy level before treatment and dyssomnia score $(\mathrm{r}=0.658, \mathrm{P}<0.001)$. There was also a positive correlation between the Hcy level after treatment and dyssomnia score $(r=0.670, P<0.001)$. Donepezil can effectively improve the sleep function of patients and reduce the Hcy level in serum in the treatment of AD patients. The application of donepezil was of great significance in the clinical treatment of AD patients.
\end{abstract}

Correspondence to: Dr Jiewen Zhang, Department of Neurology, Zhengzhou University People's Hospital, 7 Weiwu Road, Zhengzhou, Henan 450003, P.R. China

E-mail: j2q358@163.com

Key words: donepezil, Alzheimer's disease, Hcy, dyssomnia, correlation analysis

\section{Introduction}

Alzheimer's disease (AD) is the most common of all dementias, reaching approximately $70 \%$ of the total number of dementia cases (1). Dementia is characterized by impaired memory, abnormal behavior and slow thinking, due to the death of nerve cells in the brain or their inability to function normally (2). With the development of the disease, memory, self-care and motor ability decline, and emotionally continue to increase until death (3). According to statistics, there were approximately 35 million AD patients in the world in 2010, and the number of it was expected to double in 20 years (4). The disease characteristic of AD is sporadic, and only $5 \%$ of patients is caused by genetic mutations; it has the characteristic of early onset, patients usually fall ill before the age of 65 , and individual cases even fall ill at the age of 30 (5). It is very urgent for drugs, that are able to effectively treat AD, to come onto the market, because of its high incidence, poor quality of life of patients, high cost of nursing treatment and long nursing cycle (6).

It has been reported that high homocysteine (Hcy) is involved in the occurrence and development of $\mathrm{AD}$ and correlated with mental diseases worldwide. The high Hcy level can cause damage to vascular endothelial cells and vascular wall structure and cause blood clotting or form thrombosis, thus leading to cardiovascular and cerebrovascular disease and dementia. It is possible that the toxic effect caused by the product of blocked Hcy methylation leads to behavioral and psychological symptoms (7-9).

Donepezil hydrochloride is an acetylcholinesterase inhibitor with reversibility and relative specificity (10). Donepezil hydrochloride can effectively inhibit acetylcholine degradation in the central nervous system, increase acetylcholine concentration in the synaptic space of nerve cells, improve the nerve conduction function and enhance the transmission of information in the brain, thus improving the learning and memory function of the brain, having good tolerance and clinical safety (11). Numerous studies have shown that folic acid and vitamin B12, which can effectively reduce the Hcy level, and are important measures to prevent and treat high Hcy (12).

The purpose of this study was to analyze the effect of donepezil on the Hcy level in serum of AD patients and the correlation between Hcy and dyssomnia, which has important significance in clinical treatment and provides clues for the prognosis of $\mathrm{AD}$ patients. 


\section{Patients and methods}

General information. A retrospective analysis was performed and 124 AD patients in Zhengzhou University People's Hospital (Zhengzhou, China) between January 2015 and October 2017 were selected as study subjects, including 64 cases in the observation group and 60 cases in the control group, and there was no significant difference in basic indexes between the two groups ( $\mathrm{P}>0.05)$ (Table I). The control group was treated with folic acid, vitamin B12 and memantine hydrochloride tablet, and the observation group combined with donepezil on this basis, and both groups were treated for 4 months. The mean age of all patients was $65.04 \pm 10.81$ years, including 74 males and 50 females. All study subjects were diagnosed by imaging and biochemical indexes and in accordance with AD-related diagnostic criteria (13). Mental diseases were excluded such as brain dysfunction and depression and diseases combined with coronary heart disease, cerebral thrombosis and liver and kidney insufficiency were also excluded. The clinical information of all the candidates was complete, and patients or their family members signed the informed consent. General information is provided in Table I.

The study was approved by the Ethics Committee of Zhengzhou University People's Hospital (Zhengzhou, China).

Reagents and instruments. ELISA kit (cat. no. m1024045) was purchased from Shanghai Enzyme-linked Biotechnology Co., Ltd. (Shanghai, China); Antus PHOMO automatic enzymatic marker from Shanghai Sentient Life Science Development Co., Ltd. (Shanghai, China); donepezil hydrochloride tablet from Wei Wood Pharmaceutical Co., Ltd., guo yao zhun zi H20070181; folic acid from Tianjin Lisheng Pharmaceutical Co., Ltd. (Tianjin, China), guo yao zhun zi H12020215; vitamin B12 from Shanxi Yunpeng Pharmaceutical Co., Ltd. (Linfen, China), guo yao zhun zi H31023025; memantine hydrochloride tablet from Zhuhai Federal Pharmaceutical Co., Ltd. (Hong Kong, China) guo yao zhun zi H20130086.

Treatment methods. All study subjects had a light diet such as low-sugar, low-fat, and high-fiber, and maintained proper exercise and ensured adequate sleep. The observation group was treated with donepezil hydrochloride tablet combined with folic acid, vitamin B12 and memantine hydrochloride tablet. Donepezil hydrochloride tablet was used 1 time/day and $5 \mathrm{mg} / \mathrm{time}$; folic acid 1 time/day and $10 \mathrm{mg} /$ time; vitamin B12 1 time/day and $500 \mu \mathrm{g} /$ time; memantine hydrochloride tablet was used 1 time/day and $5 \mathrm{mg} /$ time in the first 3 week, and increased by $5 \mathrm{mg}$ each time from the 4 th week to $20 \mathrm{mg} /$ time and 1 time/day. The control group was treated with folic acid, vitamin B12 and memantine hydrochloride tablet, and the method was the same as that of the observation group. Both groups were treated for 4 months.

Detection of Hcy. A total of $3 \mathrm{ml}$ fasting peripheral venous blood were taken from the patients in the observation and control groups in the morning before and after 4 months of treatment, and were centrifuged at $2,600 \mathrm{x}$ g for $8 \mathrm{~min}$ at $4^{\circ} \mathrm{C}$. The serum was used for the detection of the Hcy level using ELISA method. The specific method of operation was strictly in accordance with the protocol.
Table I. General information (n, \%).

\begin{tabular}{lcccc}
\hline Factors & $\begin{array}{c}\text { Observation } \\
\text { group } \\
(\mathrm{n}=64)\end{array}$ & $\begin{array}{c}\text { Control } \\
\text { group } \\
(\mathrm{n}=60)\end{array}$ & $\chi^{2}$ & P-value \\
\hline $\begin{array}{l}\text { Age (years) } \\
\geq 65\end{array}$ & $52(81.25)$ & $50(83.33)$ & 0.817 & 0.092 \\
$<65$ & $12(18.75)$ & $10(16.67)$ & & \\
$\begin{array}{l}\text { Sex } \\
\text { Male }\end{array}$ & $35(54.69)$ & $39(65.00)$ & 0.275 & 1.369 \\
Female & $29(45.31)$ & $21(35.00)$ & &
\end{tabular}

Course of

disease (years)

$\begin{array}{lllll}\geq 2 & 48(75.00) & 43(71.67) & 0.690 & 0.176 \\ <2 & 16(25.00) & 17(28.33) & & \end{array}$

Mean Hamilton

depression scale score

$\begin{array}{lllll}\geq 12 & 38(59.38) & 40(66.67) & 0.459 & 0.706 \\ <12 & 26(40.62) & 20(33.33) & & \end{array}$

Mean simple mental

examination score

$\begin{array}{llrll}\geq 13 & 33(51.56) & 29(48.33) & 0.857 & 0.129 \\ <13 & 31(48.44) & 31(51.67) & & \\ \text { Bereft } & & & & \\ \text { Yes } & 50(78.13) & 53(88.33) & 0.155 & 2.294 \\ \text { No } & 14(21.87) & 7(11.67) & & \\ \text { Live alone } & & & & \\ \text { Yes } & 43(67.19) & 39(65.00) & 0.851 & 0.066 \\ \text { No } & 21(32.81) & 21(35.00) & & \end{array}$

Blood pressure

(mmHg)

$\begin{array}{lllll}\text { Systolic pressure } \quad 130.24 \pm 8.24 & 132.58 \pm 9.15 & 1.498 & 0.137\end{array}$

Diastolic pressure $\quad 84.35 \pm 6.25 \quad 82.07 \pm 6.81 \quad 1.944 \quad 0.054$

$\begin{array}{llllll}\beta \text {-amyloid }(\mathrm{ng} / \mathrm{ml}) & 2.17 \pm 1.61 & 2.26 \pm 1.04 & 0.367 & 0.714\end{array}$

$\begin{array}{lllll}\text { Cystine protease } & 1.06 \pm 0.08 & 1.07 \pm 0.06 & 0.783 & 0.435\end{array}$

inhibitor C $(\mathrm{mg} / \mathrm{l})$

$\begin{array}{lllll}\text { Blood sugar }(\mathrm{mmol} / \mathrm{l}) & 5.73 \pm 0.94 & 5.64 \pm 1.08 & 0.496 & 0.621\end{array}$

Dyssomnia score. The Pittsburgh Sleep Quality Index (PSQI) was used for the diagnosis of dyssomnia. The PSQI score criteria were 0-6 points for no dyssomnia, 7-11 points for mild dyssomnia, 12-16 points for moderate dyssomnia and 17-21 points for severe dyssomnia (14).

Statistical analysis. SPSS 17.0 statistical software was used for analysis (SPSS Inc., Chicago, IL, USA). ANOVA and Dunnett's test were used for comparison between multiple groups. Chi-square test was used for enumeration data. The correlation analysis between the Hcy level in serum and dyssomnia was performed using Pearson's correlation analysis. $\mathrm{P}<0.05$ was considered to indicate a statistically significant difference.

\section{Results}

Changes in the Hcy level before and after treatment. The differences were statistically significant in the Hcy level before 


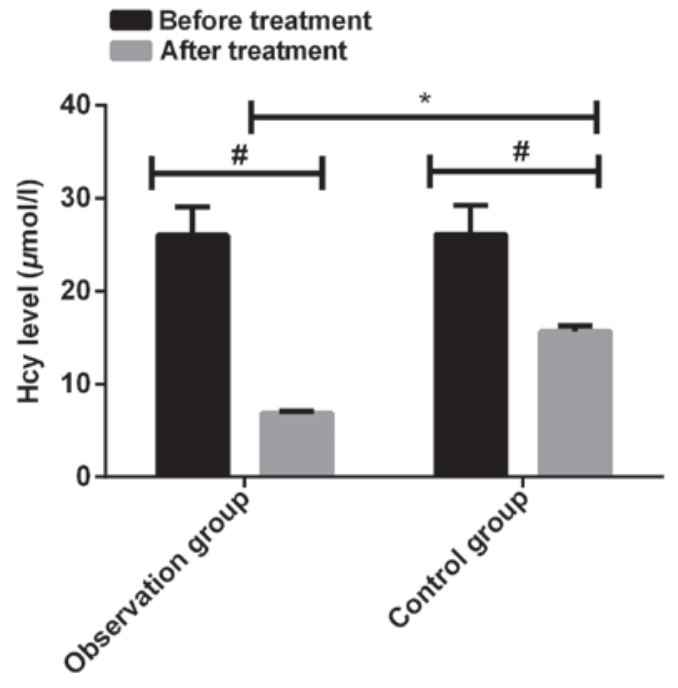

Figure 1. Changes in the Hcy level before and after treatment in the study and control groups. Results of ELISA showed that the differences were statistically significant in the Hcy level before and after treatment between the observation and control groups; ${ }^{*} \mathrm{P}<0.001$. The difference was not statistically significant in the Hcy level before treatment between the observation and control groups $(P>0.05)$. The difference was statistically significant in the Hcy level after treatment between the observation and control groups; ${ }^{*} \mathrm{P}<0.001$. Hcy, homocysteine.

Table II. Changes in the Hcy level before and after treatment.

\begin{tabular}{lccccc}
\hline Groups & $\begin{array}{c}\text { No. of } \\
\text { cases }\end{array}$ & $\begin{array}{c}\text { Treatment } \\
\text { time }\end{array}$ & $\begin{array}{c}\text { Hcy } \\
(\mu \mathrm{mol} / \mathrm{l})\end{array}$ & t value & P-value \\
\hline Observation & 64 & Before & $26.02 \pm 3.05$ & 40.70 & $<0.001$ \\
& & After & $6.86 \pm 2.21^{\text {a }}$ & & \\
Control & 60 & Before & $26.12 \pm 3.11$ & 25.54 & $<0.001$ \\
& & After & $15.65 \pm 0.64$ & & \\
\hline
\end{tabular}

${ }^{\mathrm{a}} \mathrm{P}<0.001$, compared to the Hcy level after treatment in the control group. Hcy, homocysteine.

and after treatment between the observation and control groups $(\mathrm{P}<0.001)$. The difference was not statistically significant in the Hcy level before treatment between the observation and control groups $(\mathrm{P}>0.05)$. The difference was statistically significant in the Hcy level after treatment between the observation and control groups $(\mathrm{P}<0.001)$ (Fig. 1; Table II).

Comparison of dyssomnia score before and after treatment in $A D$ patients in the observation group. The dyssomnia score before treatment was $8.99 \pm 1.43$ points, and that after treatment was $4.83 \pm 1.27$ points in the observation group. The difference was statistically significant between before and after treatment in the observation group $(\mathrm{P}<0.001)$ (Fig. 2; Table III).

Correlation analysis between the Hcy level before and after treatment and dyssomnia in the observation group. There was a positive correlation between the Hcy level before treatment and dyssomnia score $(\mathrm{r}=0.658, \mathrm{P}<0.001)$. There was a positive correlation between the Hcy level after treatment and dyssomnia score ( $r=0.670, \mathrm{P}<0.001)$ (Fig. 3; Table IV).

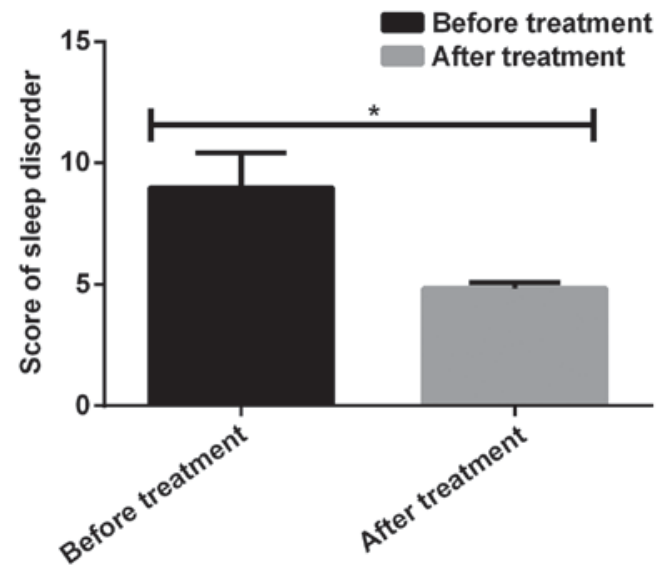

Figure 2. Comparison of dyssomnia score before and after treatment in $\mathrm{AD}$ patients in the observation group. The difference was statistically significant in dyssomnia score between before and after treatment; ${ }^{*} \mathrm{P}<0.001$. AD, Alzheimer's disease.

Table III. Comparison of dyssomnia score before and after treatment in $\mathrm{AD}$ patients in the observation group.

\begin{tabular}{lcccc}
\hline Groups & $\begin{array}{c}\text { No. of } \\
\text { cases }\end{array}$ & $\begin{array}{c}\text { Dyssomnia } \\
\text { score }\end{array}$ & t value & P-value \\
\hline Before treatment & 64 & $8.99 \pm 1.43$ & 16.81 & $<0.001$ \\
After treatment & 64 & $4.83 \pm 1.27$ & & \\
\hline
\end{tabular}

$\mathrm{AD}$, Alzheimer's disease.

\section{Discussion}

According to epidemiological statistics, AD ranks fourth in fatal diseases at present, second only to heart disease. Moreover, AD-related mortality has increased to 68\% (15). $\mathrm{AD}$ is the main cause of disability for the middle-aged and the elderly and their complete dependence on others (16). Reports worldwide have shown that high Hcy level is an independent risk factor for $\mathrm{AD}$ and cognitive impairment in patients, and that the AD risk is positively correlated with the Hcy level $(17,18)$. This study analyzed the effect of donepezil on the Hcy level in serum of AD patients and the correlation between Hcy and dyssomnia.

Results of this study showed that the differences were statistically significant in the Hcy level before and after treatment between the observation and control groups $(\mathrm{P}<0.001)$. The difference was not statistically significant in the Hcy level before treatment between the observation and control groups $(\mathrm{P}>0.05)$. The difference was statistically significant in the Hcy level after treatment between the observation and control groups $(\mathrm{P}<0.001)$. It suggested that the clinical symptoms improved slowly and the effect was not particularly satisfactory in the treatment of AD patients using folic acid, vitamin B12 and memantine hydrochloride tablet, while the combined use of donepezil hydrochloride can significantly reduce the Hcy level in AD patients, indicating the effectiveness of donepezil in the treatment of AD patients. The results of Feldman (19) agree with us. 

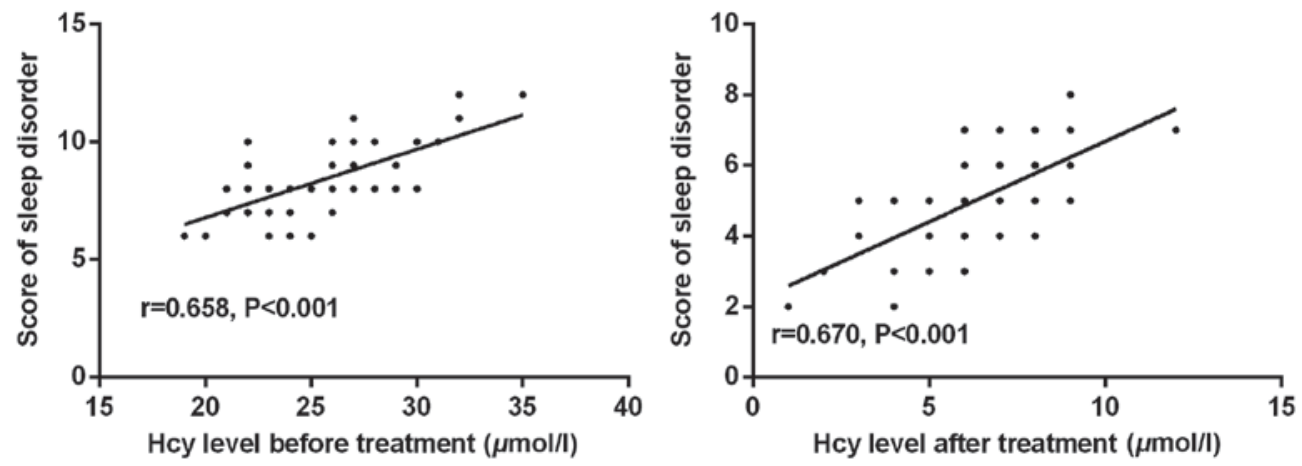

Figure 3. Correlation analysis between the Hcy level before and after treatment and dyssomnia in the observation group. Results of Pearson's correlation analysis showed that: (A) there was a positive correlation between the Hcy level before treatment and dyssomnia score $(\mathrm{r}=0.658, \mathrm{P}<0.001)$; (B) there was a positive correlation between the Hcy level after treatment and dyssomnia score $(r=0.670, \mathrm{P}<0.001)$. Hcy, homocysteine.

Table IV. Correlation analysis between the Hcy level before and after treatment and dyssomnia.

\begin{tabular}{lccccc}
\hline Groups & $\begin{array}{c}\text { Treatment } \\
\text { time }\end{array}$ & $\begin{array}{c}\text { Hcy } \\
(\mu \mathrm{mol} / \mathrm{l})\end{array}$ & $\begin{array}{c}\text { Dyssomnia } \\
\text { score }\end{array}$ & $\mathrm{r}$ & P-value \\
\hline $\begin{array}{l}\text { Observation } \\
(\mathrm{n}=64)\end{array}$ & Before & $26.02 \pm 3.05$ & $8.99 \pm 1.43$ & 0.658 & $<0.001$ \\
& After & $6.86 \pm 2.21$ & $4.83 \pm 1.27$ & 0.670 & $<0.001$
\end{tabular}

Hcy, homocysteine.

The reason for this phenomenon is the pathogenesis of $\mathrm{AD}$ is that the acetylcholine in the central nervous system that promotes the decrease of the neurotransmitter level and causes dementia symptoms. Donepezil is a cholinesterase inhibitor, and in vitro studies have shown that donepezil has a cholinesterase inhibitory effect that is several times higher than that of butyrylcholinesterase (20). Therefore, the combined use of donepezil is helpful for improvement of the sensitivity of AD treatment. In this study, the dyssomnia score before treatment was $8.99 \pm 1.43$ points and that after treatment was $4.83 \pm 1.27$ points in the observation group. The difference was statistically significant between before and after treatment $(\mathrm{P}<0.001)$. There was a positive correlation between the Hcy level before treatment and dyssomnia score $(r=0.658$, $\mathrm{P}<0.001$ ); and there was also a positive correlation between the Hcy level after treatment and dyssomnia score $(r=0.670$, $\mathrm{P}<0.001)$. The results of Werder (21) are similar to ours. He believes that the high Hcy level is correlated with low levels of folic acid, vitamin B12 and behavioral and psychological symptoms of $\mathrm{AD}$. The reason for this phenomenon may be: on the one hand, Hcy is transformed into homocystine in the body, which is a substance that has an excitotoxic effect on neurons; on the other hand, since the increase of the content of its own oxygen radicals promotes the formation of nitric oxide, the high concentration of Hcy is converted into neurotoxic substances and even lead to the death of neurons $(22,23)$. In addition, Hcy can significantly reduce the repair function of hippocampal neurons DNA and increase the AD risk, contributing to emotional abnormalities in patients, including dyssomnia and emotional instability (24).
However, there are some problems in this study. Because of the small sample size, the study was limited. Correlation analysis between the Hcy level before and after treatment in $\mathrm{AD}$ patients and other behavioral and psychological symptoms was not performed. Statistical analysis of the levels of folic acid, vitamin B12 and memantine hydrochloride tablet was not performed. There may be other influencing factors that still need further study.

In conclusion, donepezil can effectively improve the sleep function of patients and reduce the Hcy level in serum in the treatment of AD patients. The application of donepezil was of great significance in the clinical treatment of AD patients.

\section{Acknowledgements}

Not applicable.

\section{Funding}

No funding was received.

\section{Availability of data and materials}

The datasets used and/or analyzed during the present study are available from the corresponding author on reasonable request.

\section{Authors' contributions}

XL drafted the manuscript. XL and JZ were mainly devoted to collecting and interpreting the general data. MX and JL performed ELISA. SJ was responsible for detection of Hcy and dyssomnia score. All authors read and approved the final manuscript.

\section{Ethics approval and consent to participate}

The study was approved by the Ethics Committee of Zhengzhou University People's Hospital (Zhengzhou, China). Signed informed consents were obtained from the patients or guardians.

\section{Patient consent for publication}

Not applicable. 


\section{Competing interests}

The authors declare that they have no competing interests.

\section{References}

1. Isaac MG, Quinn R and Tabet N: Vitamin E for Alzheimer's disease and mild cognitive impairment. Cochrane Database Syst Rev (3): CD002854, 2008.

2. Association A; Alzheimer's Association: 2012 Alzheimer's disease facts and figures. Alzheimers Dement 8: 131-168, 2012.

3. Querfurth HW and LaFerla FM: Alzheimer's disease. N Engl J Med 362: 329-344, 2010

4. Hardy J and Selkoe DJ: The amyloid hypothesis of Alzheimer's disease: Progress and problems on the road to therapeutics. Science 297: 353-356, 2002.

5. Seshadri S, Beiser A, Selhub J, Jacques PF, Rosenberg IH, D'Agostino RB, Wilson PW and Wolf PA: Plasma homocysteine as a risk factor for dementia and Alzheimer's disease. N Engl J Med 346: 476-483, 2002.

6. Musiał A, Bajda M and Malawska B: Recent developments in cholinesterases inhibitors for Alzheimer's disease treatment. Curr Med Chem 14: 2654-2679, 2007.

7. Ingrosso D, Cimmino A, Perna AF, Masella L, De Santo NG, De Bonis ML, Vacca M, D'Esposito M, D'Urso M, Galletti P, et al: Folate treatment and unbalanced methylation and changes of allelic expression induced by hyperhomocysteinaemia in patients with uraemia. Lancet 361: 1693-1699, 2003.

8. Zhuo JM, Wang H and Praticò D: Is hyperhomocysteinemia an Alzheimer's disease (AD) risk factor, an AD marker, or neither? Trends Pharmacol Sci 32: 562-571, 2011.

9. Pandey SN, Vaidya AD, Vaidya RA and Talwalkar S: Hyperhomocysteinemia as a cardiovascular risk factor in Indian women: Determinants and directionality. J Assoc Physicians India 54: 769-774, 2006.

10. Fernandez HH, Friedman JH, Grace J and Beason-Hazen S: Donepezil for Huntington's disease. Mov Disord 15: 173-176, 2000.

11. Mazza M, Capuano A, Bria P and Mazza S: Ginkgo biloba and donepezil: A comparison in the treatment of Alzheimer's dementia in a randomized placebo-controlled double-blind study. Eur J Neurol 13: 981-985, 2006.

12. Sam RC, Burns PJ, Hobbs SD, Marshall T, Wilmink AB, Silverman SH and Bradbury AW: The prevalence of hyperhomocysteinemia, methylene tetrahydrofolate reductase C677T mutation, and vitamin B12 and folate deficiency in patients with chronic venous insufficiency. J Vasc Surg 38: 904-908, 2003.
13. Varma AR, Snowden JS, Lloyd JJ, Talbot PR, Mann DM and Neary D: Evaluation of the NINCDS-ADRDA criteria in the differentiation of Alzheimer's disease and frontotemporal dementia. J Neurol Neurosurg Psychiatry 66: 184-188, 1999.

14. Cole JC, Motivala SJ, Buysse DJ, Oxman MN, Levin MJ and Irwin MR: Validation of a 3-factor scoring model for the Pittsburgh sleep quality index in older adults. Sleep 29: 112-116, 2006.

15. Harrington CR: The molecular pathology of Alzheimer's disease. Neuroimaging Clin N Am 22: 11-22, vii, 2012.

16. Minoshima S, Giordani B, Berent S, Frey KA, Foster NL and Kuhl DE: Metabolic reduction in the posterior cingulate cortex in very early Alzheimer's disease. Ann Neurol 42: 85-94, 1997.

17. Doody RS, Demirovic J, Ballantyne CM, Chan W, Barber R, Powell S and Pavlik V; Texas Alzheimer's Disease Research and Care Consortium: Lipoprotein-associated phospholipase A2, homocysteine, and Alzheimer's disease. Alzheimers Dement (Amst) 1: 464-471, 2015.

18. Van Dam F and Van Gool WA: Hyperhomocysteinemia and Alzheimer's disease: A systematic review. Arch Gerontol Geriatr 48: 425-430, 2009.

19. Feldman H, Gauthier S, Hecker J, Vellas B, Subbiah P and Whalen E; Donepezil MSAD Study Investigators Group: A 24-week, randomized, double-blind study of donepezil in moderate to severe Alzheimer's disease. Neurology 57: 613-620, 2001.

20. Qian S, He L, Mak M, Han Y, Ho CY and Zuo Z: Synthesis, biological activity, and biopharmaceutical characterization of tacrine dimers as acetylcholinesterase inhibitors. Int J Pharm 477: 442-453, 2014.

21. Werder SF: Cobalamin deficiency, hyperhomocysteinemia, and dementia. Neuropsychiatr Dis Treat 6: 159-195, 2010.

22. Nabi H, Bochud M, Glaus J, Lasserre AM, Waeber G, Vollenweider P and Preisig M: Association of serum homocysteine with major depressive disorder: Results from a large population-based study. Psychoneuroendocrinology 38: 2309-2318, 2013.

23. Lominadze D, Tyagi N, Sen U, Ovechkin A and Tyagi SC: Homocysteine alters cerebral microvascular integrity and causes remodeling by antagonizing GABA-A receptor. Mol Cell Biochem 371: 89-96, 2012.

24. Dietrich-Muszalska A, Malinowska J, Olas B, Głowacki R, Bald E, Wachowicz B and Rabe-Jabłońska J: The oxidative stress may be induced by the elevated homocysteine in schizophrenic patients. Neurochem Res 37: 1057-1062, 2012. International (CC BY-NC-ND 4.0) License. 